\title{
Foreign body perforation of the duodenum
}

\begin{abstract}
Upper gastrointestinal perforation from a tooth pick is very rare. Most foreign bodies that are swallowed, quite often go through the gastrointestinal tract without any complications. Many of these perforations are however instigated by sharp objects like toothpicks or fish bones. Diagnosis of bowel perforation from these foreign bodies is often a great challenge, as most patients have non-specific symptoms. While such full thickness perforations have usually been treated operatively, minimally invasive option of managing these perforations with endoscopic procedures is possible.
\end{abstract}

Keywords: toothpick, foreign body, perforation, duodenum

\author{
Volume 9 Issue 4 - 2018 \\ Jothi Rajasekaran Murugesan,' Wun-Chung \\ Teoh, ${ }^{2}$ Sergei Fedorine ${ }^{2}$ \\ 'Department of General Surgery, Campbell town Hospital, \\ Australia \\ 2Department of Gastroenterology, Campbell town Hospital, \\ Australia
}

Correspondence: JothiRajasekaranMurugesan, Department of General Surgery, Campbelltown Hospital,Therry road, Campbelltown, NSW, Australia 2560, Tel +6/43|474326, Emailminrexels@gmail.com

Received: May 27, 2018 | Published: July 31, 2018

\section{Introduction}

Duodenal perforation from a foreign body is very rare. However toothpick ingestion can be associated with significant complications including perforation. Only 143 cases have been described between 1927 and $2017 .^{1-4}$

\section{Case presentation}

A 50year old woman presented to our hospital with a 4week history of dyspepsia and vague abdominal pain with exacerbation of symptoms and vomiting in the last 2 weeks prior to presentation. Her abdomen was soft and non tender on examination. Computerised tomography (CT) scan of the abdomen demonstrated mural thickening at the anterior wall of the duodenum and associated fat stranding. This was seen in association with an obliquely oriented hyperdense focus extending from the gastric lumen across the first part of the duodenum into segment 3 of adjacent liver with surrounding poorly defined irregular hypodensity measuring $21 \times 15 \times 16 \mathrm{~mm}$ in size, suspicious for liver abscess (Figure 1).

Endoscopy demonstrated a toothpick penetrating the first part of duodenum (Figure 2). This was manoeuvred and extracted endoscopically (Figure 3). After removal, there was drainage of pus from the tract. The patient had an uneventful post-procedural recovery after a short course of intravenous antibiotics and was discharged. Follow up CT scan 2 weeks later demonstrated complete resolution of the liver abscess.

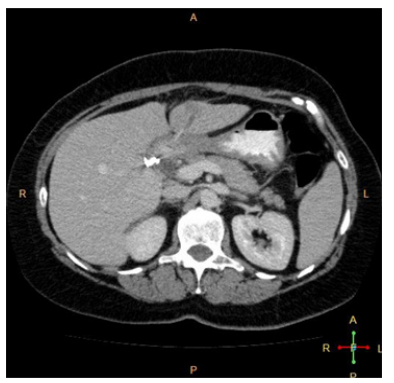

Figure I

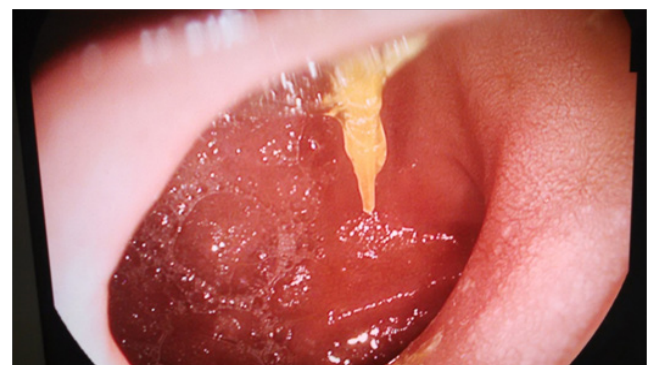

Figure 2

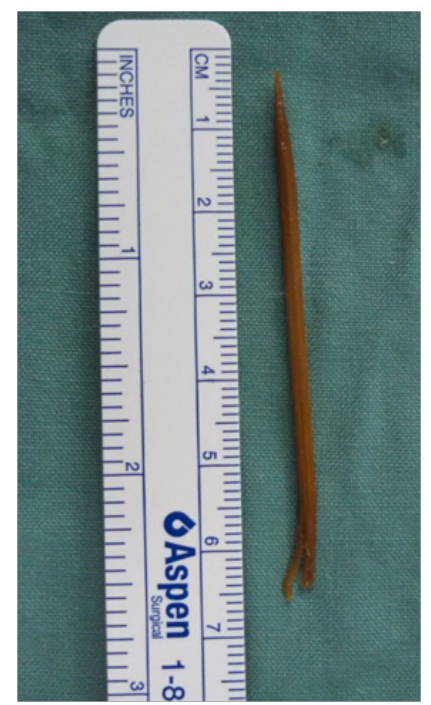

Figure 3

\section{Discussion}

Most patients are unaware of having ingested a foreign body and their presentation often differs. ${ }^{5,6} \mathrm{CT}$ scan of the abdomen is very useful in detecting foreign bodies and their associated complications. 
Most cases of perforation are caused by ingestion of thin pointed objects. ${ }^{7}$ Swallowed toothpicks very frequently lead to perforation of gastro-intestinal tract. Endoscopy is the first line management for perforation of the stomach and duodenum in patients who do not show evidence of peritonitis. ${ }^{8}$

In summary, management of swallowed toothpick can be very challenging, depending on the associated complications, as they often do not pass through and end up in perforation of the gastro-intestinal tract. Early gastroscopy with prior CT scan of the abdomen can improve the management.

\section{Acknowledgements}

None.

\section{Conflict of interest}

The author declares no conflict of interest.

\section{Patient consent}

Patient signed an informed consent and gave her permission to publish the related medical data.

\section{References}

1. Steinbach C, Stockmann M, Jara M, et al. Accidentally ingested toothpicks causing severe gastrointestinal injury: a practical guideline for diagnosis and therapy based on 136 case reports. World J Surg. 2014;38(2):371377.

2. Yang Z, Wu D, Xiong D, et al. Gastrointestinal perforation secondary to accidental ingestion of toothpicks: A series case report. Medicine (Baltimore). 2017;96(50):e9066.

3. Izumi J, Satoh K, Iwasaki W, et al. Small bowel obstruction caused by the ingestion of a wooden toothpick: the $\mathrm{CT}$ findings and a literature review. Intern Med. 2017;56(6):657-660.

4. Dorschner BW, Thouet RW, Zellweger U. Suppurativeduodenitis and superior mesenteric vein thrombosis after toothpick ingestion. Clin Gastroenterol Hepatol. 2015;13(6):A25-26.

5. St John EG. Toothpick injuries of the intestinal tract. NY State J Med. 1995;55:3115-3119.

6. Stoica M, Saftoiu A, Gheonea DI, et al. Pyogenic liver abscess caused by accidental ingestion of a wooden toothpick: role of preoperative imaging. J Gastrointest Liver Dis. 2007;16(2):221-222.

7. Cheung YC, Ng SH, Tan CF, et al. Hepatic inflammatory mass secondary to toothpick perforation of the stomach: triphasic CT appearances. Clin Imaging. 2000;24(2):93-95.

8. Singh AC, Gurney M. Toothpick penetration of stomach. Gastrointest Endosc. 2003;57(2):239 